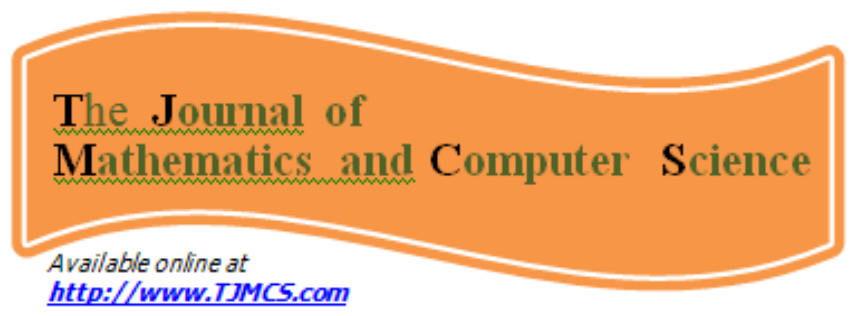

\title{
Complete derivation of the momentum equation for the second grade fluid
}

Muhammad Ayub ${ }^{1}$, Haider Zaman ${ }^{2,3}$

1. Department of Mathematics, Quaid-i-Azam University 45320,

Islamabad 44000, Pakistan

2. Department of Mathematics, Islamia College Chartered University 25120,

Peshawar 25000, Pakistan

\begin{abstract}
In a recently accepted paper of R. A. Van Gorder and K. Vajravelu [1] (Comments on "Series solution of hydromagnetic flow and heat transfer with Hall effect in a second grade fluid over a stretching sheet") Central European Journal of Physics, DOI: 10.2478/s11534-009-0145-2 (online), the authors have made momentum equation for the second grade fluid very controversial. The aim of our this communication is to give complete exact derivation of the momentum equation for second grade fluid and remedy this confusion.
\end{abstract}

PACS (2008): 47.10.A-, 47.10.ad, 47.15.Cb, 47.50.Cd, 02.70.Wz.

Keywords: Second grade fluid, Boundary layer approxmations, Thermodynamical compatibility for second grade fluid model.

\section{Introduction}

All the analysis of the second grade fluid depends strongly upon the correct form of the momentum equation. R. A. Van Gorder and K. Vajravelu [1] have presented momentum equation in following form

$$
u \frac{\partial u}{\partial x}+v \frac{\partial u}{\partial y}=\nu \frac{\partial^{2} u}{\partial y^{2}}+\lambda\left[\frac{\partial}{\partial x}\left(u \frac{\partial^{2} u}{\partial y^{2}}\right)-\frac{\partial u}{\partial y} \frac{\partial^{2} u}{\partial y^{2}}+v \frac{\partial^{3} u}{\partial y^{3}}\right] .
$$

This Eq. (1) raised the issue of a negative sign with the second term enclosed in the square brackets. For the justification of this R. A. Van Gorder and K. Vajravelu [1] have pointed out following references $[2-5]$ in their note [1]. In our dervation it comes out to be the positive sign. For justification, that our derived equation is correct, we claim that the references $[3-4]$ and $[6-11]$ supports our derived equation.

\section{Derivation}

Consider the two dimensional boundary layer flow of a non-Newtonian, incompressible and steady fluid. We assume velocity field of the form

$$
\mathbf{V}=[u(x, y), v(x, y), 0] .
$$

\footnotetext{
${ }^{3}$ Corresponding author:

Tel. : +92 03449054088

E-mail address: haiderzaman67@yahoo. com (H. Zaman)
} 
The law of conservation of mass is

$$
\frac{\partial \rho}{\partial t}+\nabla \cdot(\rho \mathbf{V})=0
$$

where $\rho$ is the density at centre of the controlled volume. For an incompressible fluid, the density is constant, with respect to space and time variables, so that Eq. (3) becomes

$$
\boldsymbol{\nabla} \cdot \mathbf{V}=0
$$

Substituting the value of $\mathbf{V}$ from Eq. (2) into the Eq. (4), the law of conservation of mass reduces to

$$
\frac{\partial u}{\partial x}+\frac{\partial v}{\partial y}=0
$$

The law of conservation of linear momentum, in mathematical form, is given by

$$
\rho \frac{d \mathbf{V}}{d t}=\operatorname{div} \boldsymbol{\sigma}+\rho \mathbf{B}_{f}
$$

where left hand side represents the inertial forces, while on the right hand side the 1st term represents surface forces while the 2 nd term $\rho \mathbf{B}_{f}$ denotes the body forces per unit volume. The body forces may be due to gravity or due to electromagnetic forces, $\boldsymbol{\sigma}$ is the Cauchy stress tensor and $\frac{d \mathbf{V}}{d t}$ is the material time derivative of the velocity $\mathbf{V}$ which can be expressed as

$$
\frac{d \mathbf{V}}{d t}=\frac{\partial \mathbf{V}}{\partial t}+(\mathbf{V} \cdot \nabla) \mathbf{V}
$$

Using Eq. (7) into Eq. (6), we get

$$
\rho\left[\frac{\partial \mathbf{V}}{\partial t}+(\mathbf{V} \cdot \boldsymbol{\nabla}) \mathbf{V}\right]=\operatorname{div} \boldsymbol{\sigma}+\rho \mathbf{B}_{f}
$$

The momentum Eq. (8) in absence of the body forces for steady incompressible fluid becomes

$$
\rho[(\mathbf{V} . \nabla) \mathbf{V}]=\operatorname{div}(\boldsymbol{\sigma}),
$$

where Cauchy stress tensor $\boldsymbol{\sigma}$ for an incompressible homogenous fluid of second grade has the form [12]

$$
\boldsymbol{\sigma}=-p \mathbf{I}+\mu \mathbf{A}_{1}+\alpha_{1} \mathbf{A}_{2}+\alpha_{2} \mathbf{A}_{1}^{2},
$$

where $\mathbf{I}$ is the identity tensor, $p$ is the pressure, $\mu$ is the coefficient of viscosity called dynamic viscosity and $\alpha_{1}, \alpha_{2}$ are the material constants. When $\alpha_{1}=0=\alpha_{2}$, the second order fluid model reduces to the classical Navier-Stokes model. The Rivilin Ericksen tensors $\mathbf{A}_{1}$ and $\mathbf{A}_{2}$ are defined as

$$
\begin{gathered}
\mathbf{A}_{1}=\mathbf{L}+\mathbf{L}^{T}, \\
\mathbf{A}_{2}=\frac{d \mathbf{A}_{1}}{d t}+\mathbf{A}_{1} \mathbf{L}+\mathbf{L}^{T} \mathbf{A}_{1},
\end{gathered}
$$

where

$$
\mathbf{L}=\operatorname{grad} \mathbf{V} \text {. }
$$

The thermodynamical compatibility for second grade fluid model were taken into account by Fosdick and Rajagopoal [13-14]. Clausius-Duhem inequality should hold together with 
the helmholtz free energy being at its minimum whenever the fluid is locally at rest. These thermodynamical constraints put restriction on the material constants:

$$
\mu \geq 0, \quad \alpha_{1} \geq 0, \quad \alpha_{1}+\alpha_{2}=0 .
$$

In component form Eq. (9) gives

$$
\begin{aligned}
& \rho\left[u \frac{\partial u}{\partial x}+v \frac{\partial u}{\partial y}\right]=\left.\operatorname{div}(\boldsymbol{\sigma})\right|_{x}, \\
& \rho\left[u \frac{\partial v}{\partial x}+v \frac{\partial v}{\partial y}\right]=\left.\operatorname{div}(\boldsymbol{\sigma})\right|_{y},
\end{aligned}
$$

As

$$
\begin{gathered}
\mathbf{L}=\operatorname{grad} \mathbf{V}=\left[\begin{array}{lll}
\frac{\partial u}{\partial x} & \frac{\partial u}{\partial v} & \frac{\partial u}{\partial z} \\
\frac{\partial v}{\partial x} & \frac{\partial v}{\partial y} & \frac{\partial v}{\partial z} \\
\frac{\partial w}{\partial x} & \frac{\partial w}{\partial y} & \frac{\partial w}{\partial z}
\end{array}\right], \\
\mathbf{L}^{T}=(\operatorname{grad} \mathbf{V})^{T}=\left[\begin{array}{llll}
\frac{\partial u}{\partial x} & \frac{\partial v}{\partial x} & \frac{\partial w}{\partial x} \\
\frac{\partial u}{\partial y} & \frac{\partial v}{\partial y} & \frac{\partial w}{\partial y} \\
\frac{\partial u}{\partial z} & \frac{\partial v}{\partial z} & \frac{\partial w}{\partial z}
\end{array}\right] .
\end{gathered}
$$

In view of velocity field given in Eq. (2),

$$
\begin{gathered}
\mathbf{A}_{1}=\mathbf{L}+\mathbf{L}^{T}=\left[\begin{array}{ccc}
2 \frac{\partial u}{\partial x} & \frac{\partial u}{\partial y}+\frac{\partial v}{\partial x} & 0 \\
\frac{\partial u}{\partial y}+\frac{\partial v}{\partial x} & 2 \frac{\partial v}{\partial y} & 0 \\
0 & 0 & 0
\end{array}\right], \\
\mathbf{A}_{1}^{2}=\left[\begin{array}{ccc}
4\left(\frac{\partial u}{\partial x}\right)^{2}+\left(\frac{\partial u}{\partial y}+\frac{\partial v}{\partial x}\right)^{2} & 2\left(\frac{\partial u}{\partial x}+\frac{\partial v}{\partial y}\right)\left(\frac{\partial u}{\partial y}+\frac{\partial v}{\partial x}\right) & 0 \\
2\left(\frac{\partial u}{\partial y}+\frac{\partial v}{\partial x}\right)\left(\frac{\partial u}{\partial x}+\frac{\partial v}{\partial y}\right) & 4\left(\frac{\partial v}{\partial y}\right)^{2}+\left(\frac{\partial u}{\partial y}+\frac{\partial v}{\partial x}\right)^{2} & 0 \\
0 & 0 & 0
\end{array}\right] .
\end{gathered}
$$

Using the Eq. of continuity (5) we have,

$$
\mathbf{A}_{1}^{2}=\left[\begin{array}{ccc}
4\left(\frac{\partial u}{\partial x}\right)^{2}+\left(\frac{\partial u}{\partial y}+\frac{\partial v}{\partial x}\right)^{2} & 0 & 0 \\
0 & 4\left(\frac{\partial v}{\partial y}\right)^{2}+\left(\frac{\partial u}{\partial y}+\frac{\partial v}{\partial x}\right)^{2} & 0 \\
0 & 0 & 0
\end{array}\right] .
$$

We have

$$
\frac{d \mathbf{A}_{1}}{d t}=\frac{\partial \mathbf{A}_{1}}{\partial t}+(\mathbf{V} \cdot \nabla) \mathbf{A}_{1},
$$

since we are dealing with the steady case, therefore, the first term vanish, so that

$$
\frac{d \mathbf{A}_{1}}{d t}=(\mathbf{V} \cdot \nabla) \mathbf{A}_{1}
$$

where

$$
(\mathbf{V} . \nabla) A_{1}=\left[\begin{array}{ccc}
2 u \frac{\partial^{2} u}{\partial x^{2}}+2 v \frac{\partial^{2} u}{\partial y \partial x} & u \frac{\partial^{2} u}{\partial x \partial y}+v \frac{\partial^{2} v}{\partial y \partial x}+u \frac{\partial^{2} v}{\partial x^{2}}+v \frac{\partial^{2} u}{\partial y^{2}} & 0 \\
u \frac{\partial^{2} u}{\partial x \partial y}+v \frac{\partial^{2} v}{\partial y \partial x}+u \frac{\partial^{2} v}{\partial x^{2}}+v \frac{\partial^{2} u}{\partial y^{2}} & 2 u \frac{\partial^{2} v}{\partial x \partial y}+2 v \frac{\partial^{2} v}{\partial y^{2}} & 0 \\
0 & 0 & 0
\end{array}\right] .
$$


Following the same pattern we get,

$$
\mathbf{A}_{2}=\left[\begin{array}{ccc}
2 u \frac{\partial^{2} u}{\partial x^{2}}+2 v \frac{\partial^{2} u}{\partial y \partial x}+4\left(\frac{\partial u}{\partial x}\right)^{2}+ & u \frac{\partial^{2} u}{\partial x \partial y}+v \frac{\partial^{2} v}{\partial y \partial x}+u \frac{\partial^{2} v}{\partial x^{2}}+ & \\
2 \frac{\partial v}{\partial x}\left(\frac{\partial u}{\partial y}+\frac{\partial v}{\partial x}\right) & v \frac{\partial^{2} u}{\partial y^{2}}+2 \frac{\partial u}{\partial x} \frac{\partial u}{\partial y}+\frac{\partial v}{\partial y}\left(\frac{\partial u}{\partial y}+\frac{\partial v}{\partial x}\right)+ & 0 \\
u \frac{\partial^{2} u}{\partial x \partial y}+v \frac{\partial^{2} v}{\partial y \partial x}+u \frac{\partial^{2} v}{\partial x^{2}}+ & \frac{\partial u}{\partial x}\left(\frac{\partial u}{\partial y}+\frac{\partial v}{\partial x}\right)+2 \frac{\partial v}{\partial x} \frac{\partial v}{\partial y} & \\
v \frac{\partial^{2} u}{\partial y^{2}}+\frac{\partial u}{\partial x}\left(\frac{\partial u}{\partial y}+\frac{\partial v}{\partial x}\right)+2 \frac{\partial v}{\partial x} \frac{\partial v}{\partial y}+ & 2 u \frac{\partial^{2} v}{\partial x \partial y}+2 v \frac{\partial^{2} v}{\partial y^{2}}+2 \frac{\partial u}{\partial y}\left(\frac{\partial u}{\partial y}+\frac{\partial v}{\partial x}\right)+ & 0 \\
2 \frac{\partial u}{\partial x} \frac{\partial u}{\partial y}+\frac{\partial v}{\partial y}\left(\frac{\partial u}{\partial y}+\frac{\partial v}{\partial x}\right) & 4\left(\frac{\partial v}{\partial y}\right)^{2} & 0
\end{array}\right] .
$$

Thus in view of the Eqs. (19), (20) and (23), the Cauchy stress tensor is given by,

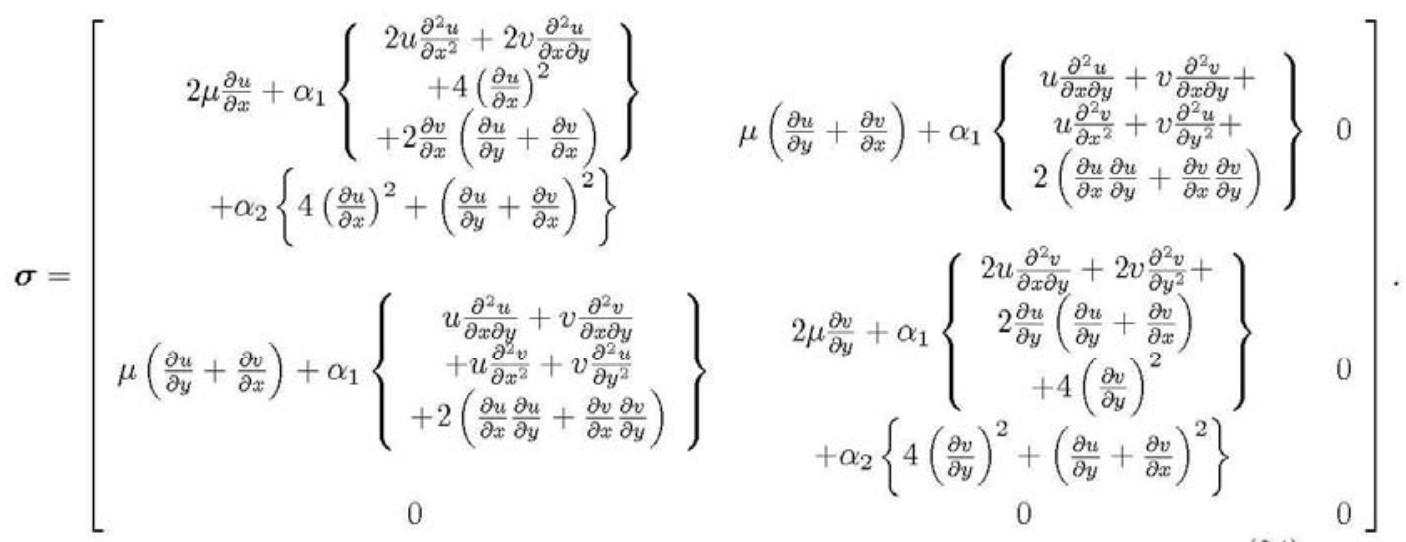

Now, we can find the divergence of $\sigma$,

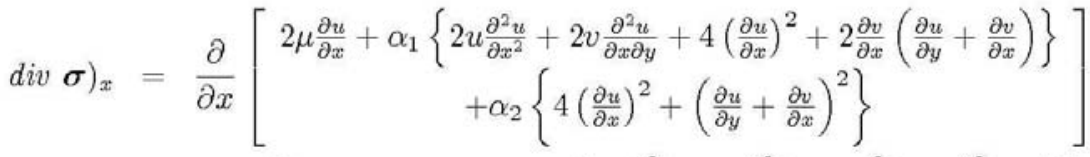

$$
\begin{aligned}
& +\frac{\partial}{\partial y}\left[\mu\left(\frac{\partial u}{\partial y}+\frac{\partial v}{\partial x}\right)+\alpha_{1}\left\{\begin{array}{c}
u \frac{\partial^{2} u}{\partial x \partial y}+v \frac{\partial^{2} v}{\partial x \partial y}+u \frac{\partial^{2} v}{\partial x^{2}}+v \frac{\partial^{2} u}{\partial y^{2}}+ \\
2\left(\frac{\partial u}{\partial x} \frac{\partial u}{\partial y}+\frac{\partial v}{\partial x} \frac{\partial v}{\partial y}\right)
\end{array}\right\}\right],
\end{aligned}
$$

using the thermodynamic model, $\alpha_{1}+\alpha_{2}=0$, i.e putting $\alpha_{2}=-\alpha_{1}$ we obtain,

$$
\begin{aligned}
d i v \boldsymbol{\sigma})_{x}= & 2 \mu \frac{\partial^{2} u}{\partial x^{2}}+\mu \frac{\partial^{2} u}{\partial y^{2}}+\mu \frac{\partial^{2} v}{\partial y \partial x}+\alpha_{1} \frac{\partial}{\partial x}\left\{\begin{array}{c}
2 u \frac{\partial^{2} u}{\partial x^{2}}+2 v \frac{\partial^{2} u}{\partial x \partial y}+4\left(\frac{\partial u}{\partial x}\right)^{2} \\
+2 \frac{\partial v}{\partial x}\left(\frac{\partial u}{\partial y}+\frac{\partial v}{\partial x}\right) \\
-4\left(\frac{\partial u}{\partial x}\right)^{2}-\left(\frac{\partial u}{\partial y}+\frac{\partial v}{\partial x}\right)^{2}
\end{array}\right\} \\
& +\alpha_{1} \frac{\partial}{\partial y}\left\{\begin{array}{c}
u \frac{\partial^{2} u}{\partial x \partial y}+v \frac{\partial^{2} v}{\partial x \partial y}+u \frac{\partial^{2} v}{\partial x^{2}}+v \frac{\partial^{2} u}{\partial y^{2}}+ \\
2\left(\frac{\partial u}{\partial x} \frac{\partial u}{\partial y}+\frac{\partial v}{\partial x} \frac{\partial v}{\partial y}\right)
\end{array}\right\} .
\end{aligned}
$$


where $\delta$ is the boundary layer thickness. The order analysis for Eq. (31) is,

$$
\begin{aligned}
& 1 \cdot \frac{1}{1}+\delta \cdot \frac{1}{\delta}=\delta^{2}\left(\frac{1}{1^{2}}+\frac{1}{\delta^{2}}\right)
\end{aligned}
$$

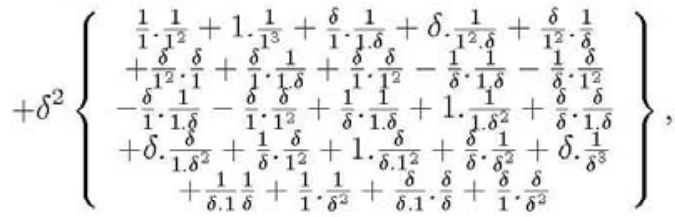

$$
\begin{aligned}
& \Rightarrow \\
& 1+1=\delta^{2}+1 \\
& +\delta^{2}\left\{\begin{array}{c}
1+1+1+1+1 \\
+\delta^{2}+1+\delta^{2}-\frac{1}{\delta^{2}}-1 \\
-1-\delta^{2}+\frac{1}{\delta^{2}}+\frac{1}{\delta^{2}}+1 \\
+1+1+1+\frac{1}{\delta^{2}}+\frac{1}{\delta^{2}} \\
+\frac{1}{\delta^{2}}+\frac{1}{\delta^{2}}+1+1
\end{array}\right\}, \\
& \Rightarrow \\
& \begin{aligned}
1+1= & \delta^{2}+1 \\
& +\left\{\begin{array}{c}
\delta^{2}+\delta^{2}+\delta^{2}+\delta^{2}+\delta^{2} \\
+\delta^{4}+\delta^{2}+\delta^{4}-1-\delta^{2} \\
-\delta^{2}-\delta^{4}+1+1+\delta^{2} \\
+\delta^{2}+\delta^{2}+\delta^{2}+1+1 \\
+1+1+\delta^{2}+\delta^{2}
\end{array}\right\},
\end{aligned}
\end{aligned}
$$

ignoring the terms of $\mathbf{O}\left(\delta^{2}\right), \mathbf{O}\left(\delta^{4}\right)$ and retaining the terms of $\mathbf{O}(1)$ then the Eq. (31) becomes,

$$
\begin{aligned}
u \frac{\partial u}{\partial x}+v \frac{\partial u}{\partial y}= & \nu \frac{\partial^{2} u}{\partial y^{2}} \\
& +\lambda\left\{\begin{array}{c}
-2 \frac{\partial u}{\partial y} \frac{\partial^{2} u}{\partial x \partial y} \\
+\frac{\partial u}{\partial y} \frac{\partial^{2} u}{\partial x \partial y}+u \frac{\partial^{3} u}{\partial x \partial y^{2}} \\
+\frac{\partial v}{\partial y} \frac{\partial^{2} u}{y^{2}}+v \partial^{3} u \\
+2 y^{3} \\
+2 \frac{\partial}{2} u^{2} u \frac{\partial u}{\partial y}+2 \frac{\partial u}{\partial x} \frac{\partial^{2} u}{\partial y^{2}}
\end{array}\right\},
\end{aligned}
$$

rearranging the terms in the brackets,

$$
\begin{gathered}
u \frac{\partial u}{\partial x}+v \frac{\partial u}{\partial y}=\nu \frac{\partial^{2} u}{\partial y^{2}}+\lambda\left\{\begin{array}{c}
\frac{\partial u}{\partial y} \frac{\partial^{2} u}{\partial x \partial y}+u \frac{\partial^{3} u}{\partial x \partial y^{2}} \\
+\frac{\partial v}{\partial y} \frac{\partial^{2} u}{\partial y^{2}}+v \partial^{2} u \\
+2 \frac{\partial u}{\partial x} \frac{\partial^{2} u}{\partial y^{2}}
\end{array}\right\}, \\
\Rightarrow \quad u \frac{\partial u}{\partial x}+v \frac{\partial u}{\partial y}=\nu \frac{\partial^{2} u}{\partial y^{2}}+\lambda\left\{\begin{array}{c}
u \frac{\partial^{3} u}{\partial x \partial y^{2}}+2 \frac{\partial u}{\partial x} \partial \partial^{2} u \\
+\frac{\partial v}{\partial y} \frac{\partial^{2} u}{\partial y^{2}}+\frac{\partial u}{\partial y} \frac{\partial^{2} u}{\partial x \partial y}+v \frac{\partial^{3} u}{\partial y^{3}}
\end{array}\right\},
\end{gathered}
$$




$$
\begin{array}{r}
\Rightarrow \quad u \frac{\partial u}{\partial x}+v \frac{\partial u}{\partial y}=\nu \frac{\partial^{2} u}{\partial y^{2}}+\lambda\left\{\begin{array}{c}
u \frac{\partial^{3} u}{\partial x \partial y^{2}}+\frac{\partial^{2} u}{\partial y^{2}}\left(2 \frac{\partial u}{\partial x}+\frac{\partial v}{\partial y}\right) \\
+\frac{\partial u}{\partial y} \frac{\partial^{2} u}{\partial x \partial y}+v \frac{\partial^{3} u}{\partial y^{3}}
\end{array}\right\}, \\
\Rightarrow \quad u \frac{\partial u}{\partial x}+v \frac{\partial u}{\partial y}=\nu \frac{\partial^{2} u}{\partial y^{2}}+\lambda\left\{\begin{array}{c}
u \frac{\partial^{3} u}{\partial x \partial y^{2}}+\frac{\partial^{2} u}{\partial y^{2}}\left(\frac{\partial u}{\partial x}+\frac{\partial u}{\partial x}+\frac{\partial v}{\partial y}\right) \\
+\frac{\partial_{u}}{\partial y} \frac{\partial^{2} u}{\partial x \partial y}+v \frac{\partial^{3} u}{\partial y^{3}}
\end{array}\right\},
\end{array}
$$

Using Eq. (5), we get,

$$
\begin{gathered}
u \frac{\partial u}{\partial x}+v \frac{\partial u}{\partial y}=\nu \frac{\partial^{2} u}{\partial y^{2}}+\lambda\left\{\begin{array}{c}
u \frac{\partial^{3} u}{\partial x \partial y^{2}}+\frac{\partial^{2} u}{\partial y^{2}}\left(\frac{\partial u}{\partial x}+0\right) \\
+\frac{\partial u}{\partial y} \frac{\partial^{2} u}{\partial x \partial y}+v \frac{\partial^{3} u}{\partial y^{3}}
\end{array}\right\}, \\
\Rightarrow \quad u \frac{\partial u}{\partial x}+v \frac{\partial u}{\partial y}=\nu \frac{\partial^{2} u}{\partial y^{2}}+\lambda\left\{\begin{array}{c}
u \frac{\partial^{3} u}{\partial \partial y^{2}}+\frac{\partial^{2} u}{\partial y^{2}} \frac{\partial u}{\partial x} \\
+\frac{\partial u}{\partial y} \frac{\partial^{2} u}{\partial x \partial y}+v \frac{\partial^{3} u}{\partial y^{3}}
\end{array}\right\}, \\
\Rightarrow \quad u \frac{\partial u}{\partial x}+v \frac{\partial u}{\partial y}=\nu \frac{\partial^{2} u}{\partial y^{2}}+\lambda\left\{\begin{array}{c}
\frac{\partial}{\partial x}\left(u \frac{\partial^{2} u}{\partial y^{2}}\right) \\
+\frac{\partial u}{\partial y} \frac{\partial^{2} u}{\partial x \partial y}+v \frac{\partial^{3} u}{\partial y^{3}}
\end{array}\right\}, \\
\Rightarrow \quad u \frac{\partial u}{\partial x}+v \frac{\partial u}{\partial y}=\nu \frac{\partial^{2} u}{\partial y^{2}}+\lambda\left\{\frac{\partial}{\partial x}\left(u \frac{\partial^{2} u}{\partial y^{2}}\right)+\frac{\partial u}{\partial y} \frac{\partial^{2} u}{\partial x \partial y}+v \frac{\partial^{3} u}{\partial y^{3}}\right\} .
\end{gathered}
$$

Consider the order analysis for Eq. (32),

$$
\begin{aligned}
& 1 . \frac{\delta}{1}+\delta \cdot \frac{\delta}{\delta}=\delta^{2}\left(\frac{\delta}{1^{2}}+\frac{\delta}{\delta^{2}}\right) \\
& +\delta^{2}\left\{\begin{array}{c}
\frac{\delta}{\delta} \cdot \frac{\delta}{\delta^{2}}+\frac{\delta}{\delta} \cdot \frac{\delta}{1^{2}}+1 \cdot \frac{\delta}{13}+1 \cdot \frac{\delta}{\delta^{2} \cdot 1}+\delta \cdot \frac{\delta}{\delta^{3}} \\
+\delta \cdot \frac{\delta}{1^{2} \delta}+\frac{1}{\delta} \cdot \frac{\delta}{1 \cdot \delta}+\frac{\delta}{1} \cdot \frac{\delta}{1 \cdot \delta} \\
+\frac{\delta}{1} \cdot \frac{1}{\delta^{2}}-\frac{\delta}{\delta} \cdot \frac{\delta}{\delta^{2}}-\frac{1}{\delta} \cdot \frac{\delta}{1 . \delta} \\
-\frac{\delta}{1} \cdot \frac{1}{\delta^{2}}-\frac{\delta}{1} \cdot \frac{\delta}{1 . \delta}
\end{array}\right\} \\
& \Rightarrow \\
& \delta+\delta=\delta^{3}+\delta \\
& +\delta^{2}\left\{\begin{array}{c}
\frac{1}{\delta}+\delta+\delta+\frac{1}{\delta}+\frac{1}{\delta} \\
+\delta+\frac{1}{\delta}+\delta \\
+\frac{1}{\delta}-\frac{1}{\delta}-\frac{1}{\delta} \\
-\frac{1}{\delta}-\delta
\end{array}\right\} \\
& \Rightarrow \\
& \begin{aligned}
\delta+\delta= & \delta^{3}+\delta \\
& +\left\{\begin{array}{c}
\delta+\delta^{3}+\delta^{3}+\delta+\delta \\
+\delta^{3}+\delta+\delta^{3} \\
+\delta-\delta-\delta \\
-\delta-\delta^{3}
\end{array}\right\} .
\end{aligned}
\end{aligned}
$$

Since there is no term of $\mathbf{O}(1)$ so all the terms of the Eq. (32) are ignored according to the boundary layer approximations. 


\section{Final remarks}

This all justify that the correct form of the momentum equation for a second grade fluid is

$$
u \frac{\partial u}{\partial x}+v \frac{\partial u}{\partial y}=\nu \frac{\partial^{2} u}{\partial y^{2}}+\lambda\left\{\frac{\partial}{\partial x}\left(u \frac{\partial^{2} u}{\partial y^{2}}\right)+\frac{\partial u}{\partial y} \frac{\partial^{2} u}{\partial x \partial y}+v \frac{\partial^{3} u}{\partial y^{3}}\right\}
$$

and there is a positive sign with the second term enclosed in the brackets.

\section{References}

[1] R. A. Van Gorder, K. Vajravelu, Central European Journal of Physics, DOI: 10.2478/s11534-009-0145-2 (online) (2009).

[2] S. K. Khan, E. Sanjayanand, Archive of Mechanics, 56191 (2004).

[3] S. Abel, P. H. Veena, K. Rajgopal, V. K. Parvin, Int. J. Non-Linear Mech. 391067 (2004).

[4] M. S. Abel, M. M. Nandeppanavar, Comm. Non-linear Sci. Numer. Simulation. 14 (5) 2120 (2009).

[5] J. E. Dunn, K. R. Rajgopal, Int. J. of Engineering Sciences. 33689 (1995).

[6] H. Zaman, M. Ayub, Central European Journal of Physics, DOI: 10.2478/s11534-0090147-0 (online) (2009).

[7] K. Vajravelu, D. Rollins, Appl. Math. Computation. 148783 (2004).

[8] M. Sajid, T. Hayat, S. Asghar, Int. J. Heat and Mass Transfer. 501723 (2007).

[9] T. Hayat, Z. Abbas, I. Pop, Numerical Methods for Partial Differential Equations. DOI 10.1002 /num.20432.

[10] F. M. Hady, R. S. R Gorla, Acta Mech. 128201 (1998).

[11] K. Sadeghy, M. Sharifi, Int. J. Non-Linear Mech. 391265 (2004).

[12] R. S. Rivilin, J. L. Ericksen, J. Rat. Mech. Anal. 3323 (1955).

[13] R. L. Fosdick, K. R. Rajagopal, Arch. Rat. Mech. Anal. 70145 (1979).

[14] J. E. Dunn, R. L. Fosdick, Arch. Ration. Mech. Anal. 56191 (1974).

[15] H. Schlichting, K. Gersten, Boundary layer theory, 8th Edition, Springer-Verlag, Berlin Heidelberg, (2000). 